\title{
Calcification detection using convolutional neural network architectures in intravascular ultrasound images
}

\author{
Hannah Sofian ${ }^{1}$, Joel Than Chia Ming ${ }^{2}$, Suraya Muhammad ${ }^{3}$, Norliza Mohd Noor ${ }^{4}$ \\ 1,2,4 Razak Faculty of Technology and Informatics, Universiti Teknologi Malaysia, Malaysia \\ ${ }^{1}$ Universiti Kuala Lumpur Malaysian Institute Information Technology, Malaysia \\ ${ }^{3}$ Universiti Kuala Lumpur Malaysian British Malaysian Institute, Malaysia
}

\section{Article Info \\ Article history: \\ Received Jun 14, 2019 \\ Revised Sep 16, 2019 \\ Accepted Sep 30, 2019}

\section{Keywords:}

Atherosclerosis

Convolutional neural network

Coronary artery calcification

Deep learning

\begin{abstract}
Cardiovascular disease is the highest leading to death for NonCommunicable disease. Coronary artery calcification disease is part of cardiovascular disease. The built-in of the plaques and the calcification in the coronary artery inner wall make the blood vessel cross-section area narrow. The standard practice by the radiologists and medical clinical are by visual inspection to detect the calcification in the intravascular ultrasound image. Deep learning is the current image processing methods that have high potential to detect calcification analysis using convolutional neural network architecture and classifiers. To detect the absence of calcification and presence calcification on the intravascular ultrasound image, using $\mathrm{k}$-fold $=10$, we compared the three types of convolutional neural network architectures and the seven types of classifiers with the provided ground truth from MICCAI 2011. We used two types of images named as Cartesian Coordinates image and polar reconstructed coordinate image. The classifiers such as Support Vector Machine, Discriminant analysis, Ensembles and Error-Correcting Output Codes obtained the perfect result with value one for Area Under Curve and all the performance measure result, accuracy, sensitivity, specificity, positive predictive value and negative predictive value. Area Under Curve for Naïve Bayes classifier is 0.9967 and for Decision Tree classifier is 0.9994 , obtained using the polar reconstructed coordinate image for InceptionresNet-V2 architecture.
\end{abstract}

Copyright $@ 2020$ Institute of Advanced Engineering and Science. All rights reserved.

\section{Corresponding Author:}

Hannah Sofian,

Razak Faculty of Technology and Informatics,

Universiti Teknologi Malaysia, Malaysia.

Email: hannah@unikl.edu.my

\section{INTRODUCTION}

Cardiovascular disease contributes $31 \%$ of the major diseases in the world according to the World Health Organization (WHO). Around $85 \%$ of the highest percentage of death in the world is caused by heart disease and stroke and major adverse cardiac events [1-2]. Coronary artery disease (CAD) is a common heart disease in the world which is also called atherosclerosis [3]. The coronary artery disease is caused by a blockage in the artery due to the development of plaque composites including calcification [2]. The calcification and the plaques developed at the artery wall made the blood volume flowing abnormally because of the area size narrowed. Calcification is part of cardiovascular disease which is called coronary artery calcification (CAC) [4]. The calcification can be detected in the intravascular ultrasound (IVUS) image when the dark artefact occurred behind the calcification. Theoretically, the wave cannot penetrate the conductor such as calcium. Therefore, IVUS modality will not measure the size area of the media boundary due to the dark shadow artefact [5]. In addition, nowadays, percutaneous coronary intervention (PCI), a nonsurgical procedure using balloon angioplasty and stent are used to open the artery vessel heart that has been 
narrowed by plaque buildup wider. However, balloon angioplasty and stent have difficulty to open the blood vessel if the calcification is presence.

The method used was three types of the convolutional neural networks $(\mathrm{CNN})$ architecture that has different size of images to detect the calcification using two types of images named the Cartesian coordinate (CC) image and the polar reconstructed coordinate (PRC) image. The previous studies to detect calcification had used many methods and techniques to detect the calcification in medical imaging images such as intravascular ultrasound (IVUS) images, optical coherence tomography (OCT) images, computed tomography-scan (CT-Scan) images and magnetic resonance imaging (MRI) images. Most of the studies used polar reconstructed coordinate (PRC) images and Cartesian coordinate (CC) image to detect the calcification in their research. Some example of the methods of calcification detection available in the literature are using Otsu threshold, morphological operation and empirical threshold by Sofian et al. [6] and using Rayleigh mixture model (RMM), Markov random field (MRF) and the graph searching method by Gao et al. [7]. Whereas, work by Katouzian et al. [8], used the Random walk framework. The parametric deformable models and geometric deformable models were used to detect the adventitia and lumen boundaries and then the Bayesian classifier to detect calcification was used by Taki et al. [9]. Unal et al. [10] used the shape-driven to detect calcification, and the adaptive thresholding was used by Filho et al. [11] to detect the calcification. Overall, the results obtained were about $80 \%$ to $95 \%$ for sensitivity and specificity. The deep learning idea is based on an artificial neural network (ANNs). The early deep learning approached was introduced by Yan LeCun in 1998 called the LeNet to recognise documents using deep learning [12-13]. An annual competition was started since 2010, called "The ImageNet Large Scale Visual Recognition Challenge (ILSVRC)" that used 1.2 million images of 1000 classes was to report the network with less error [14]. In the year 2012, the ILSVRC 2012 was won by A. Krizhevsky et al. with AlexNet model [15]. The competition challenge is continuing every year to get better performance with a low error where the authors have to perform the tasks given. In 2014, C. Szegedy et al. developed GoogLeNet which also known as Inception-V1 [16], followed by ResNet in 2015 developed by H. Kaiming et al. [17]. In 2016, the Inception Models was developed by C. Szegedy et al. based on GoogLeNet architecture [18]. Deep learning such as AlexNet architecture, VGG19Net architecture, GoogleNet architecture and ResNet152 architecture were used previously by other researchers to detect breast cancer using mammogram images by Xi et al. [19]. ResNet-50 architecture, ResNet-101 architecture and Inception-V3 architecture were eased to detect the calcification in the coronary artery using intravascular ultrasound (IVUS) images, Sofian et al 2018 [20].

In this study, we detected the presence and the absence of the calcification by feeding two types of images, Cartesian coordinate images and transformed images from Cartesian coordinate images. Three types of CNNs model; InceptionResNet-V2 architecture, Resnet-101 architecture and AlexNet architecture together with seven types of classifiers named Support Vector Machine (SVM), Discriminant Analysis (DA), Ensembles (En), Decision Tree (DT), Naïve Bayes (NB), k-Nearest Neighbours (k-NN) and Error-Correcting Output Codes (ECOC). For performance measures validation, we used cross-validation k-fold (where k=10).

\section{RESEARCH METHOD}

\subsection{Data Used In This Study}

In this study, we used dataset B from MICCAI Challenge 2011 [21]. The dataset consists of 530 images with calcification presence, and 1645 images with calcification absence. The images were taken from 10 patients extracted from in vivo pullbacks of human coronary arteries. The imaging system used for the acquisition is Si5 equipped with $20 \mathrm{MHz}$ Eagle Eye monorail catheter. In this study, we evaluate two types of image that were the CC image as shown in Figure 1a and the PRC image as shown in Figure 1b. The PRC image is the transformed image of the $\mathrm{CC}$ image. The blue arrow shows the calcification with dark shadow artefact behind it in the $\mathrm{CC}$ image and the red arrow shows the calcification with dark shadow artefact behind it in the PRC image.

Figure 1b shows the Daugman Rubber Sheet Normalisation model was used to transform from the $\mathrm{CC}$ image to the PRC image and then, assigned each point of the tunica adventitia, the tunica media (Between tunica adventitia and tunica media), tunica intima (Between lumen and tunica media) and the lumen (The middle of IVUS image). The pair coordinates unit is the real coordinate ( $r, \theta$ ) where the unit interval, $r$ is between value zero and one $[0,1]$. The theta, $\theta$ is the angular quantity that cyclic between value zero and $2 \pi[0,2 \pi]$. The IVUS region image is: $\mathrm{I}(\mathrm{x}, \mathrm{y})$, the original Cartesian coordinates are: $(\mathrm{x}, \mathrm{y})$ and the normalised polar reconstructed coordinates are: $(r, \theta)[22]$. The coordinates IVUS boundaries were along the $\theta$ direction are $\mathrm{x} 0, \mathrm{y} 0$ and $\mathrm{x}, \mathrm{y}$. The equation is as shown below:

$$
\mathrm{I}(\mathrm{x}(\mathrm{r}, \theta)), \mathrm{y}(\mathrm{r}, \theta)) \rightarrow \mathrm{I}(\mathrm{r}, \theta)
$$


Where $x(r, \theta)$ and $y(r, \theta)$ are defined as linear combinations of both sets of the lumen boundary points $(x l(\theta)$, $\mathrm{yl}(\theta))$ and the set of media boundary points along the outer perimeter of the tunica media $(\operatorname{xm}(\theta), \operatorname{ym}(\theta))$ bordering the tunica adventitia as shown below:

$$
\begin{aligned}
& x(r \cdot \theta)=(1-r) x_{l}(\theta)+r x_{m}(\theta) \\
& y(r \cdot \theta)=(1-r) y_{l}(\theta)+r y_{m}(\theta)
\end{aligned}
$$

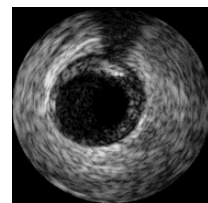

(a)

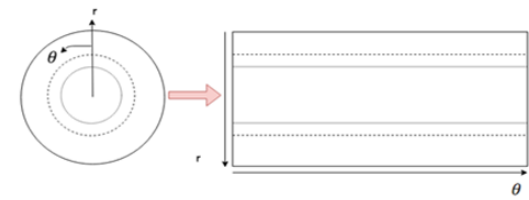

(b)

Figure 1. (a) The Cartesian coordinate (CC) image, (b) The Daugman Rubber Sheet Normalisation model with radius,r and angle, $\theta$, (c) The polar reconstructed coordinate (PRC) image

\subsection{The Series Network and Direct Acyclic Graph Network}

A series network is a convolutional layer for deep learning network where the layers were arranged from one after the other with a single input layer and a single output layer. The first CNNs types were AlexNet architecture, a series network as shown in Figure 2. The image input size used is $227 \times 227 \times 3$ based on AlexNet architecture requirement. The AlexNet architecture consists of five convolutional layers, Conv.1, Conv.-2, Conv.-3, Conv.-4 and Conv.-5 with built-in ReLu that is the deep feature extraction and three fully connected, fc6, fc7 and fc8 as shown in Figure 3. The output used was fully connected-8, fc8 layer with 1000 classes. We fed the input with two types of images that were CC image and PRC image to the AlexNet architecture and classified the result using seven types of the classifier and compared with the provided ground truth. A directed acyclic graph (DAG) network is a convolutional layers arrangement in complex architectures with multiple input layers and multiple outputs layers. The second and the third CNNs architecture were DAG Network that has a multi-input layer and a multi-output layer. Two types of DAG CNNs models were ResNet-101 architecture as shown in Figure 3 and InceptionResNet-V2 architecture as shown in Figure 4. The input image size for the residual learning network, ResNet-101 architecture is $224 \times 224 \times 3$ [17]. For the ResNet-101 architecture shown in Figure 3, we fed the two types of images that are a CC image and a PRC image. Resnet101 used bottleneck building block using three layers as shown at conv.2, conv.3, conv.4 and conv.5. The output layer is a fully connected; fc1000 layer.

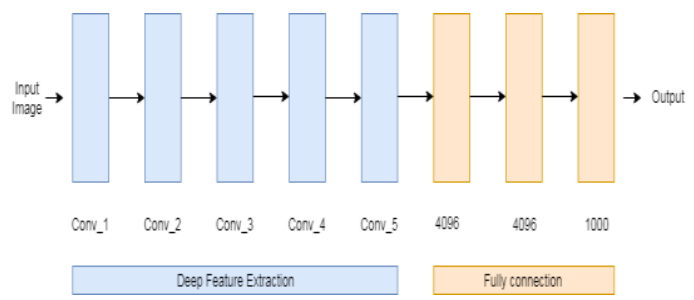

Figure 2. AlexNet block diagram

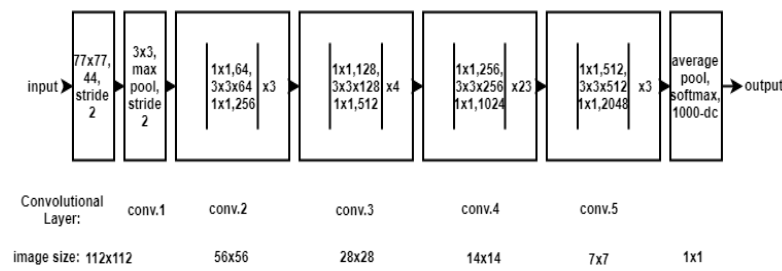

Figure 3. ResNet101 block diagram

InceptionResnet-V2 architecture is a combination architecture of the Inception model and the ResNet model. InceptionResNet-V2 architecture input image size is 299x299x3 same as Inception architecture [23]. Figure 4 shows the architecture of InceptionResnet-V2 [24]. The preprocessing image will be fed into the input size 299x229x3 as InceptionResNet-V2 architecture requirement. We used two types of images that were the CC image and PRC image as shown in Figure 1a and Figure 1b. The output layer is the fully connected, prediction layer. Figure 5 shows the block diagram used in Figure 4 for InceptionResnet-V2. In Figure 4, InceptionResNet-V2 architecture, three types of modules were used that are Inception-ResNet-A, Inception-ResNet-B and Inception-ResNet-C. InceptionResNet-V2 was inspired by combining both SqueezeNet architecture, ResNet architectures and Inception-V4 architecture. In Figure 4 shows after Stem, 
the five modules of Inception-ResNet-A was used before Reduction A block diagram, then after Reduction-A, ten modules of Inception-ResNet-B was used before Reduction B block diagram and finally before average pooling, five modules of Inception-ResNet-C was used after Reduction B block diagram. The output layer is a fully connected; prediction layer. All the ResNet architectures used residual connection but for InceptionResNet-V2, the residual connection was replaced with pooling operation. All the modules added with a $1 \times 1$ convolutional layer ( $1 \times 1$ conv) as shown in each of the modules before adding ( + ). They used a set of filters such as $1 \times 1,1 \times 3,3 \times 1,3 \times 3,5 \times 5,1 \times 7$ and $7 \times 1$ and merged in the modules.

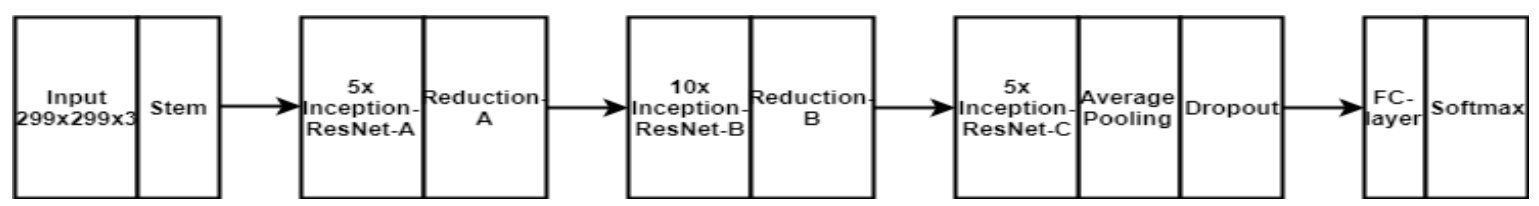

Figure 4. The InceptionResnet-V2 Architecture Block Diagram

\subsection{The Proposed Approach}

The proposed block diagram framework is shown in Figure 5. The proposed method has five steps. In the pre-processing step, the image such as shown in Figure 1a and Figure 1c will be resized to fit the CNNs architecture requirement to $227 \times 227,224 \times 224$ and $299 \times 299$ respectively. The images will be of 3input channels (RGB). In the second step, the images will be fed into the CNNs architecture i.e. AlexNet architecture, ResNet-101architecture and InceptionResNet-V2 architecture for the deep feature learning process. The output layer is the fully connected layers that were the fc8 layer for AlexNet architecture, fc1000 layer for ResNet-101 architecture and prediction layer for InceptionResNet-V2 architecture. Then, the result will be normalized to get all the output in the positive values. In the third step, classification with cross-validation will be performed. All seven types of classifier mentioned earlier is used. For the crossvalidation, the $\mathrm{k}$-fold will be divided into two parts that were training, $\mathrm{Tr}=(\mathrm{k}-1) / \mathrm{k}$ and training, $\mathrm{Te}=1 / \mathrm{k}$.

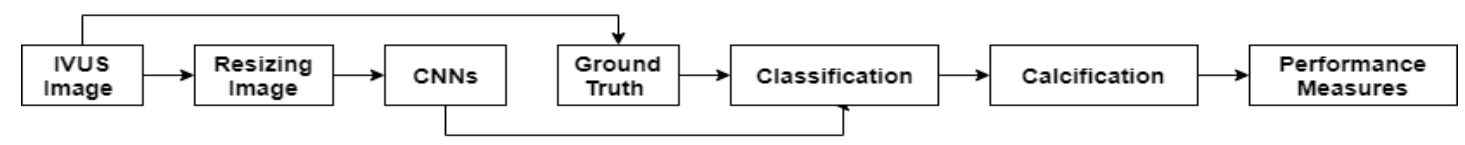

Figure 5. The calcification detection block diagram framework

\subsection{Performance Measure (PM)}

The training classifier of dataset B will be assigned with labels one, ' 1 ' and zero, ' 0 '. The label ' 1 'is referring to the calcification presence and label ' 0 ' is referring to the calcification absent. In the final step, the ground truth provided by the MICCAI Challenge 2011 will be compared to the results obtained. The ground truth validation was done by four of the clinical expert. They were blinded to the other physicians' annotations, two of them repeated the manual annotations after about one week from the first labelling. The calcification detection performance measure evaluation used is the Acc, the Sp, the Sn, PPV and NPV. The Confusion Matrix consists of the True Positive (TP), the True Negative (TN), the False Positive (FP) and the False Negative (FN) [6]. The true positive is the total of the artery disease IVUS images with calcification presence when the system was able to categorise the artery disease with calcification present. The false positive is the total of artery disease with calcification absence but was recognised with calcification present. The true negative was the total of artery disease with calcification absence when the system was able to categorise the artery disease with calcification absent. The false negative is the total of the artery disease with calcification presence but was detected as with calcification absent. Five types of performance measures are derived from the Confusion Matrix, that is Sensitivity (Sn), Specificity (Sp), Accuracy (Acc), Positive Predictive Value (PPV) and Negative Predictive Value (NPV) [18]. The Accuracy (Acc) is all of the correct prediction, TP and TN then divided by the total images in dataset $\mathrm{B}$. The highest accuracy rate is one, and the lowest rate is zero, $\mathrm{Acc}=(\mathrm{TP}+\mathrm{TN}) /(\mathrm{TP}+\mathrm{TN}+\mathrm{FP}+\mathrm{FN}))$. Sensitivity $(\mathrm{SE})$, recall (REC) or true positive rate (TPR) is the true positive (TP) then divided by the total positives number, $\mathrm{Sn}=\mathrm{TP} /(\mathrm{TP}+\mathrm{FN})$. Specificity (SP) also called true negative rate, (TNR) is the number of correct negative predictions divided by all the total negatives, $\mathrm{Sp}=\mathrm{TN} /(\mathrm{TN}+\mathrm{FP})$. Positive Prediction Value, (PPV) is the 
correct positive divided by all the positives, $\mathrm{PPV}=\mathrm{TP} /(\mathrm{TP}+\mathrm{FP})$. Negative Prediction Value, (NPV) is the correct negative divided by all the total negatives, $\mathrm{NPV}=\mathrm{TN} /(\mathrm{TN}+\mathrm{FN})[20]$.

\section{RESULTS AND ANALYSIS}

\subsection{Cartesian Coordinate (CC) Image}

Classifiers such as SVM, DA, ECOC, En and KNN performance measure tested is equal one except for DT and NB. Table 1 shows the DT classifier had Acc, Sn, Sp, PPV and NPV less than 1 (0.9737-0.9330), they are within the range of $0.9-1.0$, which are still excellent results whilst NB classifier shows that the excellent performance measure results in the NPV, the fair performance measure results in PPV whilst the Acc, the Sn and the Sp show just good performance measure results. Table 2 shows the result of ResNet-101 Architecture. Similar to AlexNet architecture, mostly all the classifiers had shown excellent performance results with all the performance measure tested equal to ' 1 ' except for classifiers such as DT and NB. Again similar to AlexNet architecture, with ResNet-101 architecture, the DT classifier performance measure for all criteria tested are in the range of $0.9774-0.9964$, which are still excellent results. NB classifier shows the excellent performance measure results in the Sn and the NPV, the fair performance measure results in PPV whilst the Acc and the Sp shows good performance measure results. Compared to the AlexNet architecture, the performance measure of NB for ResNet-101 is better.

The result for InceptionResNet-V2 Architecture is similar to ResNet-101 Architecture and AlexNet architecture, mostly all the classifiers had shown excellent performance results with all performance measure tested equal to ' 1 ' except for classifier such as DT and NB as shown in Table 3. The DT classifier performance measure for all criteria tested are in the range of $0.9794-0.9933$, which are still excellent results. NB classifier shows excellent performance measure results in the Sn, the NPV, the Acc and the Sp. However, the performance measure results show a good in PPV. Compared to, using AlexNet architecture, ResNet-101 architecture and InceptionResNet-V2 architecture, for NB classifier, ResNet-101 Architecture is better. In Table 4 to Table 6 , show the performance measure results with NB classifier when using k-fold $=2$, 3,5 and 10 for AlexNet architecture, ResNet-101 architecture and InceptionResNet-V2 architecture respectively. From Table 4, we can see that for the AlexNet architecture, $\mathrm{k}$-fold $=3$ shows good results compared to $\mathrm{k}$-fold $=2,5$ and 10 . The $\mathrm{k}$-fold $=10$ had better results compared to $\mathrm{k}$-fold $=2$. The lowest performance measure results among the $\mathrm{k}$-fold are $\mathrm{k}$-fold $=5$. Table 5 shows that the performance measures results of NB classifier and ResNet-101 architecture show that k-fold $=5$ had good results compared to kfold $=2,5$ and 10. Table 6 shows the results for NB classifier and InceptionResNet-V2 architecture. K-fold $=$ 3 had good results compared to k-fold $=2,5$ and 10 in term of the Acc, the Sn and the NPV. However, the results for the Sp and the PPV were highest with $\mathrm{k}$-fold $=2$.

Table 1. PM for AlexNet (CC)

\begin{tabular}{llllll}
\hline Classifier & Acc & Sn & Sp & PPV & NPV \\
\hline DT & 0.9885 & 0.9792 & 0.9915 & 0.9737 & 0.9933 \\
NB & 0.8294 & 0.8208 & 0.8322 & 0.6118 & 0.9351 \\
\hline
\end{tabular}

Table 3. PM for InceptionResNet-V2 (CC)

\begin{tabular}{llllll}
\hline Classifier & Acc & Sn & Sp & PPV & NPV \\
\hline DT & 0.9917 & 0.9868 & 0.9933 & 0.9794 & 0.9957 \\
NB & 0.8772 & 0.9358 & 0.8584 & 0.6804 & 0.9765 \\
\hline
\end{tabular}

Table 5. ResNet-101 with NB Classifier (CC)

\begin{tabular}{llllll}
\hline Classifier & Acc & Sn & Sp & PPV & NPV \\
\hline $\mathrm{k}=2$ & 0.8920 & 0.9377 & 0.8772 & 0.7110 & 0.9776 \\
$\mathrm{k}=3$ & 0.8947 & 0.9358 & 0.8815 & 0.7178 & 0.9771 \\
$\mathrm{k}=5$ & 0.9094 & 0.9547 & 0.8948 & 0.7452 & 0.9840 \\
$\mathrm{k}=10$ & 0.8933 & 0.9302 & 0.8815 & 0.7166 & 0.9751 \\
\hline
\end{tabular}

Table 2. PM for ResNet-101 (CC)

\begin{tabular}{llllll}
\hline Classifier & Acc & Sn & Sp & PPV & NPV \\
\hline DT & 0.9917 & 0.9774 & 0.9964 & 0.9885 & 0.9927 \\
NB & 0.8933 & 0.9302 & 0.8815 & 0.7166 & 0.9751 \\
\hline
\end{tabular}

Table 4. AlexNet with NB Classifier (CC)

\begin{tabular}{llllll}
\hline k-fold & Acc & Sn & Sp & PPV & NPV \\
\hline $\mathrm{k}=2$ & 0.8234 & 0.8019 & 0.8304 & 0.6037 & 0.9286 \\
$\mathrm{k}=3$ & 0.8405 & 0.8491 & 0.8377 & 0.6276 & 0.9451 \\
$\mathrm{k}=5$ & 0.8170 & 0.7981 & 0.8231 & 0.5924 & 0.9268 \\
$\mathrm{k}=10$ & 0.8294 & 0.8208 & 0.8322 & 0.6118 & 0.9351 \\
\hline
\end{tabular}

Table 6. InceptionResNet-V2, NB Classifier

\begin{tabular}{llllll}
\hline k-fold & Acc & Sn & Sp & PPV & NPV \\
\hline $\mathrm{k}=2$ & 0.8823 & 0.9170 & 0.8711 & 0.6963 & 0.9702 \\
$\mathrm{k}=3$ & 0.8883 & 0.9623 & 0.8644 & 0.6958 & 0.9861 \\
$\mathrm{k}=5$ & 0.8768 & 0.9472 & 0.8541 & 0.6765 & 0.9805 \\
$\mathrm{k}=10$ & 0.8772 & 0.9358 & 0.8584 & 0.6804 & 0.9765 \\
\hline
\end{tabular}

\subsection{Polar Reconstructed Coordinate (PRC) Image}

The results show that our proposed method had an excellent result when taken $\mathrm{k}$-fold $=10$. Table 7 to Table 9 show the performance measure results when using polar reconstructed coordinate (PRC) image for the AlexNet architecture, ResNet-101 architecture and InceptionResNet-V2 architecture using the seven 
classifiers mentioned earlier. In addition, Table 10 to Table 12 show the results of NB classifier using the CC image for the k-fold=2, 3, 5 and 10. Table 7 shows mostly all the of classifiers had shown excellent performance measure results with the all performance measure tested equal to ' 1 ' except for DT and NB. The DT performance measure for all criteria tested is in the range of $0.9682-0.9867$, which are still excellent results. However, for NB classifier shows that the excellent performance measure results in the Sn and the NPV, the fair performance measure results in PPV whilst the ACC and the Sp shows just good performance measure results. Table 8 also shows that most all the classifiers had excellent performance measure results with all performance measure tested equal to ' 1 ' except for DT classifier and NB classifier. The DT classifier performance measure for all criteria tested is in the range of $0.9520-0.9914$, which are still excellent results. However, for NB classifier shows that the excellent performance measure results in the Sn and the NPV, the fair performance measure results in PPV whilst the Acc and the Sp show just good performance measure results. Table 9 also shows that most all the classifiers had excellent performance results with all the performance measure tested equal to ' 1 ' except classifiers for DT, k-NN and NB. The DT classifier performance measure for all criteria tested are in the range of $0.9962-1.0000$, and $\mathrm{k}-\mathrm{NN}$ classifier performance measure is between $0.9984-1.0000$, which are still excellent results. The result for NB classifier also improves with InceptionResNet-V2 architecture and PRC image. The performances measure are in the range of $0.8863-1.0000$. Table 10 to Table 12 shows the performance measure results with NB classifier when using $\mathrm{k}$-fold $=2,3,5$ and 10 for AlexNet architecture, ResNet-101 architecture and InceptionResNet-V2 architecture. Table 10 shows that $\mathrm{k}$-fold $=3$ had good results compared to $\mathrm{k}$-fold $=25$ and 10 except for NPV, the lowest results among the NPV. Table 11 shows that $\mathrm{k}$-fold $=2$ had good results compared to $\mathrm{k}$-fold $=3,5$ and $10 . \mathrm{K}$-fold $=10$ has the lowest performance measures results $\mathrm{k}$-fold among all. $\mathrm{K}$-fold $=5$ performance measure results are better than $\mathrm{k}$-fold $=3$. Table 12 shows that $\mathrm{k}$-fold $=2$ had good results compared to $\mathrm{k}$-fold $=3,5$ and $10 . \mathrm{K}$-fold $=10$ is the lowest performance measures results among all kfold. $\mathrm{K}$-fold $=5$ performance measure results are better than $\mathrm{k}$-fold $=3$.

\subsection{Receiver Operating Characteristics (ROC) Graph and Area under Curve (AUC) Graph}

In Figure 6 shows a CC Image for ResNet101 shows excellence results compared to AlexNet and InceptionResNetV2 whilst a PRC image, AlexNet architecture, ResNet-101 architecture and InceptionResnet-V2 architecture, NB classifier (Yellow line) shows improved performance measures. DT classifier (Magenta line) had improved the performance measure result in PRC images for InceptionResNet-V2 architecture but had a better result in the CC image using AlexNet architecture and ResNet-101 architecture. Although AlexNet architecture and ResNet-101 architecture had shown improvement in the polar reconstructed coordinate image the performance measure still slightly low as compared to InceptionResNet-V2 architecture. Based on the experiment, NB classifier had the lowest result among other classifiers tested for AUC when using k-fold $=10$. Figure 6 shows the AUC using NB classifier using CC images and PRC images. When compared between CC image and PRC image, ResNet-101 show an excellent result for DT and NB classifier whilst InceptionResNetV2 shows an excellent result for DT and NB classifier. Among Figure 6, AUC with InceptionResNet-V2 architecture using the PRC image is the highest. The threshold near to the y-axis and same value with the top line that is one [25].

Table 7. PM for AlexNet (PRC)

\begin{tabular}{llllll}
\hline Classifier & Acc & Sn & Sp & PPV & NPV \\
\hline DT & 0.9867 & 0.9774 & 0.9897 & 0.9682 & 0.9927 \\
NB & 0.8699 & 0.9019 & 0.8596 & 0.6742 & 0.9645
\end{tabular}

Table 9. PM for InceptionResNet-V2 (PRC)

\begin{tabular}{llllll}
\hline Classifier & Acc & Sn & Sp & PPV & NPV \\
\hline DT & 0.9917 & 0.9868 & 0.9933 & 0.9794 & 0.9957 \\
NB & 0.9687 & 1.0000 & 0.9587 & 0.8863 & 1.0000 \\
\hline
\end{tabular}

Table 11. ResNet-101 with NB Classifier

\begin{tabular}{llllll}
\hline Classifier & Acc & Sn & Sp & PPV & NPV \\
\hline $\mathrm{k}=2$ & 0.9669 & 0.9943 & 0.9581 & 0.8842 & 0.9981 \\
$\mathrm{k}=3$ & 0.9605 & 0.9868 & 0.9520 & 0.8688 & 0.9955 \\
$\mathrm{k}=5$ & 0.9623 & 0.9906 & 0.9532 & 0.8721 & 0.9968 \\
$\mathrm{k}=10$ & 0.8699 & 0.9019 & 0.8596 & 0.6742 & 0.9645 \\
\hline
\end{tabular}

Table 8. PM for ResNet-101 (PRC)

\begin{tabular}{llllll}
\hline Classifier & Acc & Sn & Sp & PPV & NPV \\
\hline DT & 0.9816 & 0.9736 & 0.9842 & 0.9520 & 0.9914 \\
NB & 0.8699 & 0.9019 & 0.8596 & 0.6742 & 0.9645 \\
\hline
\end{tabular}

Table 10. AlexNet with NB Classifier

\begin{tabular}{llllll}
\hline k-fold & Acc & Sn & Sp & PPV & NPV \\
\hline $\mathrm{k}=2$ & 0.8611 & 0.8679 & 0.8590 & 0.6647 & 0.9671 \\
$\mathrm{k}=3$ & 0.8887 & 0.9377 & 0.8729 & 0.7040 & 0.9528 \\
$\mathrm{k}=5$ & 0.8713 & 0.9094 & 0.8590 & 0.6751 & 0.9775 \\
$\mathrm{k}=10$ & 0.8699 & 0.9019 & 0.8596 & 0.6742 & 0.9645 \\
\hline
\end{tabular}

Table 12. InceptionResNet-V2, NB Classifier

\begin{tabular}{llllll}
\hline k-fold & Acc & Sn & Sp & PPV & NPV \\
\hline $\mathrm{k}=2$ & 0.9940 & 1.0000 & 0.9921 & 0.9761 & 1.0000 \\
$\mathrm{k}=3$ & 0.9779 & 1.0000 & 0.9708 & 0.9170 & 1.0000 \\
$\mathrm{k}=5$ & 0.9798 & 1.0000 & 0.9733 & 0.9233 & 1.0000 \\
$\mathrm{k}=10$ & 0.9687 & 1.0000 & 0.9587 & 0.8863 & 1.0000 \\
\hline
\end{tabular}




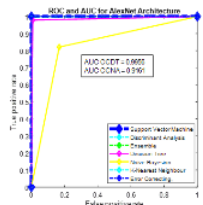

(a) AlexNet

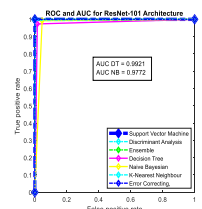

(b) ResNet101

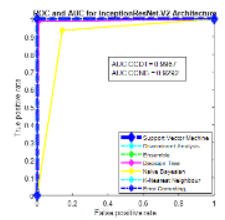

(c)InceptionRes Net-V2

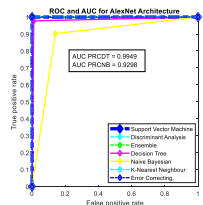

(d) AlexNet

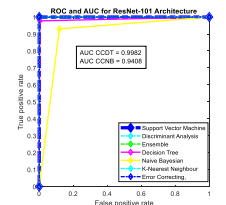

(e) ResNet-101

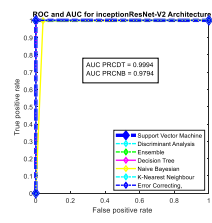

(f)InceptionRes Net-V2

Figure 6. Receiver operating characteristics and area under curve for $\mathrm{CC}$ image (Figure a, Figure $b$ and Figure $c$ ) and PRC Image (Figure e, Figure $f$ and Figure g)

\subsection{Naïve Bayes $k-F o l d ~(k=2,3,5$ \&10)}

For AlexNet architecture, Figure $7 \mathrm{a}$ show that the k-fold=3 in the $\mathrm{CC}$ image had better AUC value compared to $\mathrm{k}$-fold $=10, \mathrm{k}$-fold $=2$ and $\mathrm{k}$-fold $=5$ whilst and Figure $7 \mathrm{~d}$ shows that $\mathrm{k}$-fold $=5$ in PRC image had better AUC compared $\mathrm{k}$-fold $=10, \mathrm{k}$-fold $=2$ and $\mathrm{k}$-fold $=3$. The PRC image had better AUC value with 0.9298 compared to $\mathrm{CC}$ image using $\mathrm{k}$-fold $=10$. In this case, AlexNet architecture can use $\mathrm{k}$-fold $=10$ for PRC Image. For ResNet-101 architecture, Figure $7 \mathrm{~b}$ shows that the $\mathrm{k}$-fold $=10$ in the CC image had better AUC value compared to $\mathrm{k}$-fold $=2, \mathrm{k}$-fold $=5$ and $\mathrm{k}$-fold $=3$ whilst and Figure $7 \mathrm{e}$ shows that $\mathrm{k}$-fold $=3$ in PRC image had better AUC value compared $\mathrm{k}$-fold $=2$, $\mathrm{k}$-fold $=10$ and $\mathrm{k}$-fold $=5$. The PRC image had better AUC value of 0.9710 compared to $\mathrm{CC}$ image using $\mathrm{k}$-fold $=3$. For InceptionResNet-V2 architecture, Figure 7c shows that the $\mathrm{k}$-fold $=3$ using CC image had better AUC value compared to $\mathrm{k}$-fold $=10, \mathrm{k}$-fold $=$ 2 and $\mathrm{k}$-fold $=3$ whilst and Figure $7 \mathrm{f}$ shows that $\mathrm{k}$-fold $=3$ in PRC image had better AUC value compared kfold $=10, \mathrm{k}$-fold $=2$ and $\mathrm{k}$-fold $=3$. The PRC image had better AUC value with 0.9961 compared to CC image using $\mathrm{k}$-fold $=3$. In general, InceptionResNet-V2 architecture for NB classifier, with AUC $=0.9961$ gave a better accuracy and good classifier using polar reconstructed coordinate image using $\mathrm{k}$-fold $=3$ and on par with DT classifier value with AUC $=0.9994$.

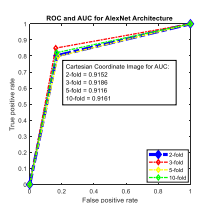

(a) AlexNet

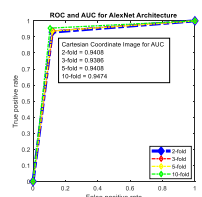

(b) ResNet-101

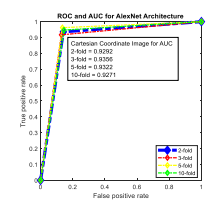

(c)InceptionRes Net-V2

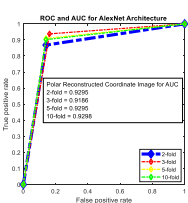

(d) AlexNet

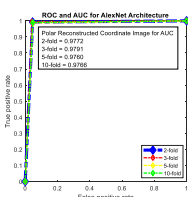

(e) ResNet-101

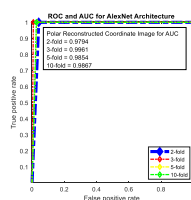

(f)InceptionRes Net-V2

Figure 7. k-fold of ROC and AUC of CC Image (Figure a, Figure b and Figure c) and PRC Image (Figure d, Figure e and Figure f) using Naïve Bayes Classifier

\section{CONCLUSION}

In this study, all classifier had obtained excellent performance measure results using a Cartesian coordinate image when using cross-validation, k-fold $=10$ with AlexNet architecture, ResNet-101 architecture and InceptionResNet-V2 architecture. As a conclusion, InceptionResNet-V2 architecture shows the excellent performance measure in Cartesian coordinate image and Polar Reconstructed coordinate to all architectures and classifier except for Naïve Bayes classifier and Decision Tree. However, the improvement in Naïve Bayes shows excellent results and almost perfect classifier using Polar Reconstructed Coordinate images. Based on the AUC value of the ROC curve, InceptionResNet-V2 shows the perfect architecture and perfect all classification using Polar Reconstructed image. For further research, classifiers such as Naïve Bayes and Decision Tree can be improved.

\section{ACKNOWLEDGEMENTS}

We would like to thank Professor Dr Simone Balocco, Computer Vision Center, Dept. Matemàtica Aplicada i Anàlisi, Bellaterra-Universitat de Barcelona, Barcelona for his cooperation in this study. The research funded by the Ministry of Higher Education Malaysia, Universiti Teknologi Malaysia Research Grant (Q.K. 130000.2540.14H22) and Universiti Kuala Lumpur (UniKL/CoRI/str16026). 


\section{REFERENCES}

[1] U. Datathe, W. Health, and S. Series, "Understanding data in the world health statistics series". 2018.

[2] M. S. Lo-Kioeng-Shioe et al., "Coronary Calcium Characteristics as Predictors of Major Adverse Cardiac Events in Symptomatic Patients: Insights From the CORE 320 Multinational Study," J. Am. Heart Assoc., vol. 8, no. 6, p. e007201, 2019.

[3] M. V. Madhavan, M. Tarigopula, G. S. Mintz, A. Maehara, G. W. Stone, and P. Généreux, "Coronary artery calcification: Pathogenesis and prognostic implications," J. Am. Coll. Cardiol., vol. 63, no. 17, pp. 1703-1714, 2014.

[4] W. Liu et al., "Current understanding of coronary artery calcification,” pp. 668-675, 2015.

[5] M. Samin K. Sharma, MD, Yuliya Vengrenyuk, PHD, Annapoorna S. Kini, "IVUS, OCT, and Coronary Artery Calcification," JACC Cardiovasc. IMAGING, vol. 10, no. 8, pp. 878-880, 2017.

[6] H. Sofian, A. Ng, J. C. M. Than, S. Mohamad, and N. MNoor, "Calcification boundary detection in coronary artery using intravascular ultrasound images," in IEEE Region 10 Annual International Conference, Proceedings/TENCON, 2017, vol. 2017-Decem, pp. 2835-2839.

[7] Z. Gao et al., "Automated Detection Framework of the Calcified Plaque with Acoustic Shadowing in IVUS Images," PLoS One, vol. 9, no. 11, 2014.

[8] A. Katouzian et al., "Iterative self-organizing atherosclerotic tissue labeling in intravascular ultrasound images and comparison with virtual histology.," IEEE Trans. Biomed. Eng., vol. 59, no. 11, pp. 3039-49, Nov. 2012.

[9] A. Taki et al., "Automatic segmentation of calcified plaques and vessel borders in IVUS images," International Journal of Computer Assisted Radiology and Surgery", vol. 3, no. 3-4. Springer, pp. 347-354, 2008.

[10] G. Unal, S. Bucher, S. Carlier, G. Slabaugh, T. Fang, and K. Tanaka, "Shape-driven segmentation of the arterial wall in intravascular ultrasound images," IEEE Trans. Inf. Technol. Biomed., vol. 12, no. 3, pp. 335-347, 2008.

[11] E. S. Filho, Y. Saijo, A. Tanaka, T. Yambe, S. Li, and M. Yoshizawa, "Automated Calcification Detection and Quantification in Intravascular Ultrasound Images by Adaptive Thresholding," IFMBE Proc., vol. 14, no. 3, pp. 1421-1425, 2007.

[12] Y. Lecun, Y. Bengio, and G. Hinton, "Deep learning," Nature, vol. 521, no. 7553. pp. 436-444, 2015.

[13] P. H. Yann LeCun, Leon Bettou, Yoshua Bengio, "Gradient-Based Applied to Recognition Document," Proceeding IEEE, pp. 1-46, 1998.

[14] O. Russakovsky et al., "ImageNet Large Scale Visual Recognition Challenge," Int. J. Comput. Vis., vol. 115, no. 3, pp. 211-252, 2015.

[15] A. Krizhevsky, I. Sutskever, and G. E. Hinton, "ImageNet Classification with Deep Convolutional Neural Networks," Adv. Neural Inf. Process. Syst., pp. 1-9, 2012.

[16] C. Szegedy, "Deep Neural Networks for Object Detection,” Nips 2013, pp. 1-9, 2013.

[17] K. He, X. Zhang, S. Ren, and J. Sun, "Deep Residual Learning for Image Recognition,” 2015.

[18] C. Szegedy, V. Vanhoucke, S. Ioffe, J. Shlens, and Z. Wojna, "Rethinking the Inception Architecture for Computer Vision," 2015.

[19] P. Xi, C. Shu, and R. Goubran, "Abnormality Detection in Mammography using Deep Convolutional Neural Networks," 2018.

[20] H. Sofian, J. C. M. Than, S. Mohammad, and N. M. Noor, "Calcification Detection of Coronary Artery Disease in Intravascular Ultrasound Image: Deep Feature Learning Approach," Int. J. Integr. Eng., vol. 10, no. 7, pp. 43-57, 2018.

[21] S. Balocco et al., "Standardized evaluation methodology and reference database for evaluating IVUS image segmentation.," Comput. Med. Imaging Graph., vol. 38, no. 2, pp. 70-90, Mar. 2014.

[22] J. Daugman, "How Iris Recognition Works."

[23] C. Szegedy, S. Ioffe, V. Vanhoucke, and A. Alemi, "Inception-v4, Inception-ResNet and the Impact of Residual Connections on Learning," pp. 4278-4284, 2016.

[24] C. Szegedy, S. Ioffe, V. Vanhoucke, and A. Alemi, “The Impact of Residual Connections on Learning,” 2016.

[25] T. Fawcett, “An introduction to ROC analysis," Pattern Recognit. Lett., vol. 27, no. 8, pp. 861-874, 2006.

\section{BIOGRAPHIES OF AUTHORS}

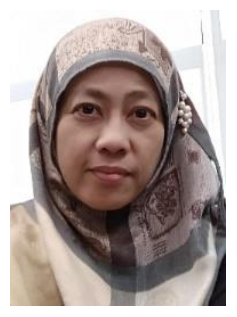

Hannah Sofian, she is currently attached as Lecturer in Universiti Kuala Lumpur Malaysian Institute Information Technology, Universiti Kuala Lumpur (UniKL), Kuala Lumpur. She received her B. Eng. in Electrical and Electronics Engineering from West of Scotland University in Paisley, Scotland and her Master of Science in Mechatronics (Signal and Systems) from International Islamic University Malaysia (IIUM) and currently her final semester in $\mathrm{PhD}$ (Electrical Engineering) from Razak Faculty of Technology and Informatics, Universiti Teknologi Malaysia (UTM). Her research interest is in the signal processing, image processing and image analysis and computer vision. Her current work concentrates on medical image analysis for coronary artery diseases in deep learning. 

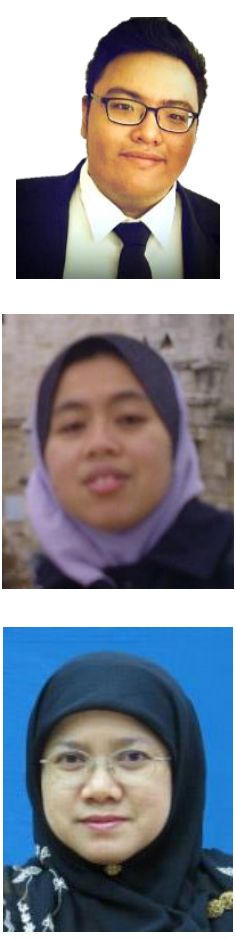

Joel Chia Ming Than, he received his $\mathrm{PhD}$ and Master of Philosophy from Universiti Teknologi Malaysia. He received B. Eng in Biomedical Engineering from Universiti Tunku Abdul Rahman. $\mathrm{He}$ is passionate is in medical imaging, machine learning and deep learning.

Dr Suraya Mohammad, she is a Senior Lecturer in Universiti Kuala Lumpur, British Malaysian Institute. She graduated with a Bachelor degree in Engineering from the University of Tasmania, Australia in 1997, completed an MSc degree in Mechatronic at the IIUM in 2006 and was awarded a PhD degree in Computer Science from The University of Manchester, UK in 2015. Her research interest is in data communication, networking and signal and image processing with application in biomedical and computer vision.

Associate Professor Dr Norliza Mohd Noor, she is currently attached as Associate Professor in Razak Faculty of Technology and Informatics, Universiti Teknologi Malaysia (UTM), Kuala Lumpur Campus. She received her B. Sc. In Electrical Engineering from Texas Tech University in Lubbock, Texas and her Master of Electrical Engineering (by research) and $\mathrm{PhD}$ (Electrical Engineering) from UTM. Her research area is in image processing and image analysis. Her current work concentrates on medical image analysis for lung diseases. She has published many papers in journals and in indexed conference proceedings and has published one academic book and two book chapters. Currently, she is the Head of the Electrophysiology Research Group, UTM Razak Faculty of Technology and Informatics. 